\title{
ENTORNOS DE APRENDIZAJE MIXTO EN EDUCACIÓN SUPERIOR
}

\section{(BLENDED LEARNING ENVIRONMENTS AT HIGHER EDUCATION)}

\author{
Antonio Bartolomé Pina \\ Universitat de Barcelona (España)
}

\section{RESUMEN}

En la segunda mitad de los años noventa irrumpió con fuerza en la Educación Superior un diseño de formación basado exclusivamente en TIC soportando entornos no presenciales: el "e-learning". Al mismo tiempo, los entornos presenciales comenzaron a incorporar esas mismas tecnologías, lo que ha derivado en el "Blended Learning".

La mayoría de diseños e-learning no se diferencian de los diseños clásicos de EaD, aunque enriquecidos con tecnología. El nuevo siglo ha traído dos líneas diferentes de evolución: una la de quienes han percibido que los diseños e-learning suponían algo más que el uso de tecnología y han comenzado a trabajar sobre nuevos diseños, por ejemplo en entornos centrados en el "contenedor", entornos abiertos, etc. La otra ha incorporado espacios de presencialidad evolucionando hacia el Blended Learning.

Se entiende por Blended Learning un sistema en el que se mezclan situaciones de presencialidad y no presencialidad, recurriendo a las tecnologías más adecuadas para cada necesidad. Este artículo repasará algunas de esas tecnologías, tratando de ofrecer las claves para un modelo general de BL.

Palabras clave: blended learning, enseñanza semipresencial, e-learning, entornos virtuales, educación virtual.

\begin{abstract}
At the second half of the past century, a new learning design appeared at Higher Education, based in technology enhanced virtual environments: e-learning. At the same time, conventional institutions adopted these ICT, generating "Blended Learning".

The most of e-learning systems do not differ of traditional ODL, but enhanced by technology. Two new lines have appeared with the new century: one is centred in developing a new model for e-learning. The other mixes F2F and virtual resources in a mixed way.
\end{abstract}


Blended Learning is defined as a system where $\mathrm{F} 2 \mathrm{~F}$ and virtual situations are mixed, based in the more adequate technology for each need. This text will review some technologies, trying to offer a new model for their implementation.

Key words: blended learning, e-learning, virtual learning environments.

Puede parecer una contradicción hablar de Blended Learning en una revista de Enseñanza a Distancia. Nada más lejos de la realidad. Aunque no aplicable a todos los casos, algunos recursos presenciales proporcionan a la $\mathrm{EaD}$ el complemento perfecto que le permite subsanar deficiencias y mejorar los resultados de la formación.

\section{ENSEÑANZA A DISTANCIA Y BLENDED LEARNING}

En un primer análisis del punto de encuentro de la Enseñanza a Distancia y el Blended Learning, debemos comenzar por precisar el segundo concepto y recordar la evolución de la primera.

\section{Blended Learning. Concepto}

El término Blended Learning (BL), que podríamos traducir como aprendizaje mixto, hace referencia al uso de recursos tecnológicos tanto presenciales como no presenciales en orden a optimizar el resultado de la formación.

He tratado detenidamente en otros lugares los conceptos Blended Learning y e-Learning (Bartolomé, 2004; Bartolomé y Aiello, 2006). Ello me excusa de extenderme, pero señalaré algunos aspectos clave.

La combinación de elementos presenciales y no presenciales es lo que caracteriza el BL (Coaten, 2003; Marsh, 2003). No es un concepto nuevo (Brodsky, 2003) ni siquiera es la única forma de nombrarlo: Marsh (2003) habla de "hybrid model".

$\mathrm{Al}$ igual que el e-learning, BL es un término que nace en el marco del "negocio" de la formación: Brennan (2004) habla de "cualquier posible combinación de un amplio rango de medios para proveer aprendizaje diseñado para resolver problemas específicos de negocio".

El término "learning" resalta el papel del estudiante al hablar de "aprendizaje" más que de "enseñanza". Esto ha recibido algunas críticas. Oliver y Trigwell (2005) 
plantean objeciones tanto desde un punto de vista filosófico como por el hecho de que, aunque el término se refiere al "aprendizaje" lo que realmente vemos detrás es el tratamiento de entornos de "enseñanza". En una línea similar Bartolomé (2004) prefiere utilizar el término "Enseñanza semipresencial" cuando hablamos del diseño de la formación: de hecho es el alumno el que convierte el "BL" no importa qué diseño (por ejemplo, reuniéndose con compañeros de un curso a distancia o preparando mediante el correo electrónico un trabajo a presentar en un curso presencial).

Entendido como un diseño formativo, los términos más utilizados en Español son:

\begin{tabular}{|l|l|}
\hline Enseñanza semipresencial & Bartolomé (2001); Leão y Bartolomé (2003) \\
\hline Educación flexible & Salinas (1999) \\
\hline Formación mixta & Pascual (2003) \\
\hline
\end{tabular}

Pero si nos situamos en un entorno de EaD es necesario retomar el concepto de BL dejando de lado la idea de diseñar un entorno semipresencial. Se trata, pues de integrar algunas herramientas tipo "face to face" en entornos básicamente a distancia. Esto no es complicado pues si bien el BL aparece en programas de formación presencial al integrar recursos tecnológicos de tipo no presencial, también ha sido analizado como una evolución del e-learning.

\section{El camino de la EaD hacia el e-learning}

La Enseñanza Abierta y a Distancia posee ya una sólida tradición. García Aretio (1999) explica que la EaD surge por una conjunción de factores:

- Los avances sociopolíticos,

- La necesidad de aprender a lo largo de la vida,

- La carestía de los sistemas convencionales,

- Los avances en el ámbito de las ciencias de la educación,

- Las transformaciones tecnológicas.

Es éste último el que sin embargo parece determinar, según el mismo autor, la evolución. Señala tres etapas y la evolución de una a otra siempre viene marcada por las transformaciones tecnológicas: 
- La enseñanza por correspondencia (la más larga).

- La enseñanza multimedia.

- La enseñaza telemática.

Sitúa el comienzo de ésta última etapa en los años ochenta pero hace notar de la existencia de una segunda subetapa que es la que coincide con la eclosión de la WWW y la aparición del término "e-learning".

Casas y Stojanovic (1999) se extienden en el análisis de la tercera etapa mencionada distinguiendo esas dos partes a las que nos referíamos, relacionándolas con las conferencias de Vancouver (1982) y Viena (1989).

Mientras en la primera se generaliza el modelo planteado por la Open University, la segunda destaca por el énfasis puesto en las nuevas tecnologías.

Y hay un aspecto especial que merece ser resaltado: "Así mismo, surgen cada vez mayores dudas sobre la futura exclusividad de esta modalidad sólo para las Universidades especializadas a Distancia" (pp. 4 de 17). Estamos ante el salto al Blended Learning.

Esto ha llevado a trabajos que describen el Blended Learning como la gran solución, como un planteamiento capaz de provocar mejores resultados en los exámenes y generar un mayor grado de satisfacción entre los estudiantes (Heterick \& Twigg, 2003, p. 28). Creo que estos estudios deben situarse en los clásicos trabajos que demuestran la bondad automática de los medios y que hace más de 20 años que fueron ampliamente relativizados (Aliaga y Bartolomé, 2006).

\section{DIFICULTADES Y PROBLEMAS}

A pesar de la presión recibida desde las instituciones y empresas que han recurrido al e-learning como argumento de venta de sus programas de formación, "en los últimos años se han levantado voces críticas que han resaltado las limitaciones del llamado e-learning” (Pascual, 2003; Bartolomé, 2004).

Más allá de una discusión estéril lo que nos interesa aquí es mostrar las debilidades del modelo e-learning para ver qué puede aportar el BL y cómo debemos plantearlo. Estas debilidades son: 
- Inadecuación del modelo formativo.

- Inadecuación de las soluciones tutoriales.

- Insuficiente consideración de los aspectos emocionales.

- Equivocada interpretación de la consistencia del entorno entendida como uniformización de los cursos.

Vamos a comentarlas en detalle.

\section{Inadecuación del modelo formativo}

Mientras el e-learning se presenta como una opción que permite atender un número mayor de estudiantes gracias a las soluciones tecnológicas, en la práctica toma el modelo clásico de la $\mathrm{EaD}$, modelo que repetidamente se ha mostrado como adecuado para un perfil preciso de sujetos que no es el mayoritario en la población (Bartolomé, 2004).

Esto se constata en el menor número de titulados que terminan su carrera. Por ejemplo, en Cataluña, si miramos la relación entre titulados que terminan y alumnos matriculados los cursos 2000-2001, 2001-2002 y 2002-2003 (últimos datos disponibles por la Sindicatura de Cuentas) vemos que, mientras para el conjunto de universidades públicas presenciales fue 0,15, para la Universitat Oberta osciló entre 0,01 y 0,03. Con otras palabras, el curso 2002-2003 la UOC sólo obtuvo 680 titulados de entre sus 21371 estudiantes (Sindicatura de Comptes de Cataluña, 2006).

Datos similares se obtienen en la mayoría de instituciones de Educación Superior a Distancia, lo que debe llevar a pensar que no es un problema de la institución en particular, sino una limitación del modelo.

\section{Un modelo para pocos sujetos}

¿Qué sujetos tienen éxito en la EaD (referida a la Educación Superior)? Son sujetos que, además de poseer habilidades de lecto-escritura (que no parecen ser tan necesarias en la enseñanza presencial), poseen una elevada capacidad de organizarse el tiempo y el trabajo, de trabajar orientados a objetivos, una cierta ventaja del estilo cognitivo de independencia de campo... Esto explica la reiterada alta mortandad de los programas de EaD. Pero ¿qué pasa en la enseñanza presencial? ¿no son necesarias esas características? 
La mayoría de los alumnos universitarios han sido educados (en la escuela básica) en una forma de aprender en la que el grupo es un entorno natural y necesario, tanto como la acción del profesor con un ritmo impuesto por el encuentro presencial en las sesiones de clase. Grupo y ritmo/contacto continuado parecen dos factores que facilitan a los alumnos que no responden al perfil indicado anteriormente, continuar sus estudios. Por supuesto, existen otros factores, como un mayor peso de la comunicación oral, elementos emocionales relacionados con la comunicación no verbal, etc.

Casas y Stojanovic (1999) describen así el modelo de EaD imperante en los años ochenta y noventa: "En Vancouver 82 la concepción principal sobre Educación a Distancia giró alrededor del modelo desarrollado con gran éxito por la Open University, (OU) de la Gran Bretaña, ... Por consiguiente, el modelo de la OU se estructuró alrededor de un texto impreso de gran calidad en su contenido y con una diagramación sumamente creativa, tanto desde el punto de vista pedagógico como estético. Este elemento, se complementó con algunos recursos audiovisuales, principalmente a través de Radio y Televisión, ... Finalmente, una red de Centros Regionales, coordinaba la compleja operación de numerosos orientadores y tutores, provenientes de una gran diversidad de instituciones que apoyaron a la Open University."

La introducción de las tecnologías no alteró el modelo. En el fondo el modelo se basa en el eje materiales-tutor, pero en el que el diseño sigue partiendo del concepto de grupo: creación de perfiles que definen el propio contenido de la formación, el cual realmente no es individualizado a cada sujeto. Es el sujeto el que se tiene que adaptar al proceso de formación.

\section{Del eje "material-tutor" al eje "entorno-sujeto"}

Es necesario trabajar sobre un nuevo modelo en el que el elemento clave deja de ser el eje material-tutor para ser el eje entorno-sujeto. El sujeto es un ser diferenciado a nivel cognitivo, metacognitivo (e.g. la reflexión sobre su propio aprendizaje) y no-cognitivo (e.g. aspectos emocionales), es decir que posee características, potencialidades y necesidades diferentes en los tres ámbitos. Es el entorno el que se debe adaptar al sujeto, respondiendo a sus necesidades de formación (diferenciales) mediante recursos y soluciones diferentes. Nótese que eso no quiere decir en absoluto que se trate de procesos individuales: el proceso de formación (como la vida) se produce en el seno de un grupo pero atendiendo a la diversidad. 
Curiosamente la $\mathrm{EaD}$ clásica funciona al revés: el proceso de aprendizaje se produce en el ámbito de la individualidad pero sin atender a la diversidad. El nuevo modelo se produce en el ámbito del grupo (que hoy llamaríamos una "red social orientada al aprendizaje") pero atendiendo a la diversidad de los individuos.

Naturalmente existen opciones diferentes: ¿quién asegura la adaptación al individuo? Algunos autores plantean que sea el entorno el que se adapte al sujeto. Un buen ejemplo de ello son los sistemas Hipermedia Adaptativos. Estos sistemas pretenden tanto adaptar la forma de presentar la información en los documentos como guiar al sujeto en su navegación por el hiperespacio (Delestre, Pécuchet y Barri-Gréboval, 1999). Otra forma de definirlos sería como sistemas hipermedia que almacenan internamente modelos de las características del usuario así como de otros elementos y los utiliza para adaptar la forma como se presenta la información (Gutiérrez y Pérez, 2001).

Creo que esa solución hace años que demostró su inviabilidad: los costos de producción de entornos adaptados son tan elevados, con escasas evidencias de que en su aplicación final vayan a ser aprovechados, como elevado es el desconocimiento que todavía tenemos sobre las diferencias individuales. Piénsese por un momento en qué elementos diferenciadores escogeríamos: ¿̇estilos cognitivos? De verdad creemos que las diferencias individuales vienen respondidas por los estilos cognitivos hoy al uso? Sin menoscabar la relevancia de, por ejemplo, la "dependencia-independencia de campo" no podemos por menos de reconocer que existen muchas variables en el sujeto que son difícilmente medibles cuando no simplemente desconocidas.

Por ello otra aproximación que hoy gana fuerza es la que se basa en capacidad del propio sujeto de autorregular su aprendizaje.

\section{Competencias para un nuevo modelo}

Una definición comúnmente aceptada de aprendizaje autorregulado es la de Schunk and Zimmerman (1998): "El aprendizaje que ocurre fundamentalmente bajo de la influencia de las ideas, sentimientos, estrategias y conductas generadas por el propio estudiante, y que están orientados a la consecución de objetivos".

Pero también podríamos definirlo como el "conjunto de competencias que permite a los estudiantes controlar las variables que tienen un impacto en su proceso de aprendizaje" (Cilia y Ot. 2006). 
No es necesario resaltar que precisamente las capacidades requeridas para desarrollar un aprendizaje autorregulado tienen mucho que ver con las requeridas en el viejo modelo de la EaD. Pero el modelo cambia en tanto en cuanto se adapta decididamente a esa competencia para autorregular el aprendizaje creando un entorno que lo facilita y lo potencia.

El modelo clásico de EaD requería de esta competencia pero al mismo tiempo la coartaba al trabajar sobre itinerarios curriculares y propuestas metodológicas impuestas desde arriba y en las que el alumno apenas podía desarrollar sus propias estrategias. En el fondo, en la EaD podemos decir que el currículum ha sido víctima del propio currículum. En la enseñanza presencial está a punto de pasar algo parecido.

Este modelo que aquí se propone requiere de nuevas competencias que deben adquirirse en la formación básica, como (entre otras):

- Autorregulación del aprendizaje (“Self-regulated learning”).

- Competencia digital tal como la describe la Comisión Europea (2005), es decir incluyendo la capacidad crítica de manejar información.

- Conocimiento de otras lenguas para acceder a otras fuentes de recursos.

Y es en el marco de ese modelo donde el recurso a soluciones presenciales (sea un seminario específico, una reunión periódica, un encuentro del grupo, ...) debe ser considerado, como una respuesta a una necesidad diferenciada.

Para una revisión de cómo el aprendizaje autorregulado es considerado en diferentes entornos de aprendizaje potenciados por las tecnologías, ver el texto de Kart Steffens (2006). Para una revisión de casos de "Comunidades de práctica" ver la revisión de Christopher Jonson (2001).

\section{Inadecuación de las soluciones tutoriales}

La EaD ha reconocido tradicionalmente la importancia de la acción tutorial. Y se ha recurrido a todas las tecnologías disponibles en cada momento para facilitar esa acción. El e-learning ha heredado la conciencia de la importancia de ese aspecto y ha tratado de aplicar las nuevas tecnologías pero sin ofrecer un modelo alternativo. 


\section{Un modelo basado en la persona del "tutor" con apoyo de materiales}

De nuevo aquí el problema es el modelo. En el modelo tradicional de EaD el tutor es una persona que, bien a distancia o en los centros distribuidos, atiende al sujeto individualmente. La tecnología adopta un claro papel mediador. Por ello se recurre fundamentalmente a tecnologías comunicativas y no informativas.

Pero si analizamos la clase tradicional veremos que tiene un carácter "tutorial" que es olvidada en los diseños de e-learning. En los entornos presenciales la acción orientadora no se realiza exclusivamente mediante la entrevista, es decir, la comunicación personal tutor-alumno. El sistema crea un modelo en el que existen diferentes recursos para responder a las necesidades del sujeto.

Cuando el e-learning no se plantea una solución global sino que basa la acción tutorial en la acción del tutor, incurre en un incremento de costos inasumible. Así existen datos (College Board, 2001) que muestra un incremento de los costos de "tuition" del 5,5\% al 7,7\%, alcanzando el curso 2003-2004 un incremento del 14\%.

Los sistemas de e-learning han buscado dos "soluciones-parche" a este problema: la contratación de tutores de bajo costo y el recurso a soluciones automáticas (agentes inteligentes tutores).

Mientras que la segunda opción no termina de mostrar resultados satisfactorios con el carácter que se le pretende dar (sustituto del tutor humano), la primera se ha traducido en un descenso de la calidad de la enseñanza.

Así prestigiosas instituciones de formación a distancia han ido forzando a sus tutores a multiplicar por 10 y por 20 el número de alumnos que debían atender, en el mismo número de horas, o mejor dicho, con el mismo salario. Los tutores, forzados a atender más alumnos, recurren a una mayor dedicación (no remunerada), una lectura más superficial de los mensajes, un uso más generalizado de soluciones genéricas no siempre adecuadas a las necesidades del alumno, incremento del retraso en la respuesta lo que a su vez le genera ansiedad y estrés que también afecta a la calidad de su trabajo,... además de no disponer de tiempo para leer y actualizarse, descuidando su propia formación continua. 


\section{La sobreexplotación del tutor}

Mientras unas pocas instituciones mantienen en sus programas de formación a distancia la contratación como tutores de profesores expertos (con la consiguiente repercusión en los costes y en los precios) son muchas las que, para atender a grupos cada vez más numerosos, recurren a alguna de estas soluciones, siempre en la línea de tutores de bajo costo:

- Profesionales a quienes no les importa que su trabajo no esté remunerado económicamente de una forma justa, ya que encuentran satisfactorio el título de "profesor universitario" que reciben. Para ellos es su actividad profesional la que les proporciona los recursos económicos para vivir. Esta solución es quizás una de las mejores.

- Académicos con contratos de bajo nivel (tiempo parcial) en instituciones presenciales. Estos académicos pueden beneficiarse en ocasiones de los recursos que su institución les ofrece, de modo que su actividad como tutor a distancia en otras instituciones representa un sobresueldo. Esta solución puede implicar una pérdida de calidad en su dedicación a la institución donde imparte clases presenciales y no suele ser muy bien vista por sus dirigentes.

- Académicos recién graduados que no encuentran oportunidades de trabajo mejor.

En los últimos años he visto el recurso a académicos de países en situación económica más precaria, para quienes el salario bajo de una institución de un país más rico se convierte en un buen salario en su propio país. También en ocasiones académicos-emigrantes. ¿Es poco creíble un escenario en el que instituciones potentes y prestigiosas del Norte ofrezcan sus programas a distancia a estudiantes del "Sur", para los que contraten "tutores del Sur" que ejerzan desde el propio "Sur" y cobren "salarios del Sur"? ¿Terminarán los estudiantes latinoamericanos pagando un plus que puede representar un $400 \%$ de incremento de costes, por recibir la misma formación y con los mismos tutores, pero eso sí, recibiendo una pomposa titulación en Inglés?

También he visto a auténticos profesionales de la tutoría, capaces de atender cursos en instituciones diferentes saltando de una a otra de un modo similar a la anécdota de un profesor de Educación Física que era capaz de dar simultáneamente clase en tres centros diferentes, por el procedimiento de dejar a unos alumnos jugando a un deporte mientras se desplazaba para atender a otro grupo. 
Vistos todos juntos el panorama parece desolador. Por supuesto, esta no es la situación en todos los casos. Es preferible que cada uno revise honradamente las situaciones que conoce y haga su propio análisis.

De la persona "tutor" al entorno "tutor"

De nuevo aquí se hace necesario repensar el modelo. Dejemos de pensar por un momento en la figura del tutor y pensemos en un entorno, parte del entorno de aprendizaje global, en el que se produce la acción tutorial: un entorno tutorial.

Este entorno está formado por diferentes recursos que ayudan al alumno a resolver sus dificultades, y a orientar su aprendizaje. Esta es una lista de algunos de estos recursos:

- FAQ. Listas de preguntas frecuentes que el alumno puede consultar de modo "anónimo" y donde frecuentemente descubre nuevas dudas de las que ni siquiera era consciente.

- Ayuda online sensible al contexto. En el momento, la aclaración a un punto.

- Guías del alumno: para comenzar a trabajar, para alumnos "noveles", guías que le permiten introducirse progresivamente en el entorno y en la materia.

- Listas de discusión donde no sólo aprenden los que preguntan, sino también los alumnos que contestan. Y un mirador privilegiado para el profesor que puede descubrir errores no sospechados.

- Sesiones de Chat: la agilidad del diálogo para temas que permiten un tratamiento sencillo y directo.

- Correo electrónico asistido, una instancia más cuando otros recursos fallan y se hace preciso superar barreras de espacio y tiempo.

- Sistemas inteligentes, más como una opción de futuro, que como una realidad, pero que también ofrecen soluciones en entornos muy precisos.

- Entrevistas vía videoconferencia de despacho. La entrevista personal por encima de la distancia, y reduciendo la pérdida de aspectos emocionales de la comunicación. 
- Entrevistas personales, un entorno privilegiado para el encuentro y la tutoría.

- Reuniones en grupo con el profesor: la optimización del tiempo con otras ventajas añadidas.

En realidad es todo el entorno el que actúa de modo que en el modelo que he presentado sujeto-entorno la acción tutorial no se entiende como un elemento aparte del proceso sino un elemento integrado en la propia autorregulación. De ese modo cualquier actividad puede tener un carácter tutorial.

\section{Insuficiente consideración de los aspectos emocionales}

Es frecuente encontrar sistemas e-learning que prescinden total o en gran medida de los aspectos emocionales. Su importancia ha sido objeto de discusión y estudio desde hace años. Pascual (2003) señala: “...la ausencia de contacto humano dificulta sentirse parte de una comunidad educativa, el elevado grado de motivación necesaria para seguir un curso on-line".

En la enseñanza presencial los aspectos emocionales están presentes de modo natural a través del uso de lenguajes no verbales: una frase del profesor siempre incluye un aspecto emocional pues siempre la acompaña una expresión facial, un tono, una posición corporal.

\section{El audiovisual como lenguaje emocional}

En la EaD esta dimensión emocional debe ser tratada por ejemplo de modo explícito, cuidando el texto de los mensajes electrónicos, o mediante el recurso a medios audiovisuales, como videoconferencias, en los que es posible introducir alguno de estos elementos.

De ahí la importancia que adquiere el audiovisual en los cursos de e-learning. En mi opinión esta es una de las dos vías de trabajo con las que estos cursos deben afrontar el problema de la carencia emocional de sus cursos. Fíjense por ejemplo como la introducción de la imagen del profesor mejora esta dimensión en los materiales polimedia de la Universidad Politécnica de Valencia: http://polimedia. upv.es/polimedia/index.asp 


\section{El encuentro personal complementario a la comunicación a distancia}

Pero existe otra vía en la que de nuevo los planteamientos de BL nos aportan soluciones. Repasemos un momento nuestras sensaciones cuando leemos un texto y comparemos esas sensaciones cuando conocemos personalmente al autor y cuando no lo conocemos. Veremos como inmediatamente la percepción del texto varía: el texto actúa como un revividor de experiencias anteriores y acoge emociones ligadas a dichas experiencias.

De ahí la importancia de que incluso en los sistemas a distancia, los alumnos puedan asociar a sus compañeros o tutores con personas de carne y hueso con quienes han convivido y han llegado a apreciar.

De ahí se deduce que las ocasiones en que podemos juntar profesores y alumnos en un curso a distancia, como por ejemplo, para un seminario o un taller, no deben organizarse exclusivamente, ni diría que principalmente, en relación al aprendizaje de competencias sino en el marco del desarrollo de ese entorno de aprendizaje (virtual en gran parte pero presencial en ese momento), aportando aquellos elementos que la presencialidad precisamente puede aportar.

Y eso es precisamente BL: escoger el recurso más adecuado de acuerdo a sus potencialidades y a las necesidades del sujeto.

\section{Equivocada interpretación de la consistencia del entorno entendida como uniformización de los cursos}

Existe una cierta obsesión de las instituciones por homogeneizar los cursos a distancia. Técnicamente podríamos estar hablando de consistencia del entorno, aunque seguramente se hablará más de imagen de marca o de la necesidad del alumno de trabajar de modo similar en todos los cursos.

Esto es tanto más sorprendente cuando precisamente se adoptan soluciones que separan el contenido de la presentación (xml). Pero vayamos un momento a una institución presencial.

\section{Un modelo tradicional en la EaD}

En la Educación Superior, que es de lo que trata este artículo, no lo olvidemos, se ha hablado tradicionalmente de la libertad de cátedra. Defendida o denostada, 
lo que creo que es ampliamente aceptado es que los alumnos no tienen ninguna dificultad en trabajar con diferentes profesores, diferentes metodologías, diferentes planteamientos. Algunos profesores prefieren explicar con ejemplos, otros utilizan PowerPoint, hay quien llena la pizarra, mientras los hay que hablan sin recurrir a ningún medio. $Y$ de nuestra propia experiencia como estudiantes podemos recordar que hemos encontrado profesores buenos y malos en todos estos y otros casos. Y también que hemos sabido trabajar con todos ellos. ¿Por qué tiene que ser un problema que los profesores de entornos virtuales utilicen diferentes recursos?

Cuando las instituciones pretenden que los profesores utilicen todos la misma plataforma, con cursos diseñados de la misma manera y con los mismos recursos no parecen tener en cuenta la diferencia entre los cursos que pretenden desarrollar competencias de tipo más práctico y los que se mueven en un ámbito más teórico y reflexivo.

Pero la situación todavía es peor cuando se defienden sistemas en los que la presentación y organización de los cursos es la misma en todos los casos, y el profesor-diseñador se convierte en un "rellenador" de bases de datos con contenidos, que luego son presentados de modo estándar. No sólo se descuidan los aspectos diferenciales de la formación por lo que respecta a los alumnos, sino que también se olvidan por lo que se refiere a los profesores y a las materias.

Conviene destacar la barbaridad que encierra este modelo: en vez de entender que diseñar un curso es diseñar un entorno de aprendizaje, pensando las actividades más adecuadas para alcanzar los objetivos o desarrollar las competencias deseadas, lo que hacemos es un único modelo con recursos estándar (léase un foro, un calendario, etc.) que se supone válido para todos los cursos, todos las materias, todos los profesores y todos los alumnos, y el diseño específico del curso no es sino colocar contenidos: estamos confundiendo "formar" con "informar".

\section{Hacia un modelo flexible}

El primer elemento para superar estas deficiencias es dar mayor peso al profesortutor, en definitiva al responsable que está en contacto más directo con los alumnos. Diferentes profesores recurrirán a diferentes técnicas, estrategias, métodos... Y por supuesto a diferentes tecnologías. 
Un marco común proporcionará consistencia al entorno. Pero los alumnos se encontrarán delante de profesores-tutores que son, para empezar, personas, y como tales, diferentes unos de otros.

Un aspecto clave de este modelo es la posibilidad de utilizar herramientas no presentes en el entorno. Es frecuente hoy que la primera pregunta que se plantea los responsables de un sistema formativo que va a implementar un "Tele" "Technology enhanced learning environment" o "entorno de aprendizaje potenciado por la tecnología”) es qué plataforma utilizar. Esta es una pregunta interesante pero que no debe condicionar en absoluto el diseño pedagógico del entorno. Y esto por dos hechos que parecen olvidarse:

- Contra lo que podría suponerse, casi con cualquier plataforma es posible plantear casi cualquier diseño pedagógico. Las plataformas tecnológicas están hechas para ser supeditadas al proceso de formación, forzadas si es necesario. Por ello es un terrible error que sean los responsables de informática quienes tomen decisiones en este punto. El modo como se implementa la plataforma debe estar en manos de los didactas con conocimientos suficientes de tecnología y con el apoyo de los técnicos en computadoras.

- Y, como es fácil de suponer, NINGUNA plataforma resuelve TODOS los problemas. Pero esto no es una dificultad si sabemos como trabajar con herramientas abiertas en Internet e integrarlas en la plataforma.

Es en el marco de ese modelo flexible donde introduciremos recursos presenciales según convenga.

\section{Las personas no son tornillos}

No puedo terminar este apartado sin insistir en este comentario que, no por obvio, parece menos olvidado: las personas no son tornillos. Existe desde hace años una tendencia a plantear el diseño de los programas de formación en términos de "optimización", “uniformización", “estandarización”, etc. Es más, me temo que si analizamos los procesos de evaluación de la calidad de la enseñanza encontraremos más de lo mismo.

Si fabrico tornillos supongo que puedo llegar a determinar cuál es el ángulo preciso, el movimiento exacto, la duración de un gesto que permite obtener un mayor número de tornillos sin defectos. 
Pero en el aprendizaje de las personas la situación no es la misma. Un mismo foro, un mismo recurso, un mismo ejercicio, una misma actividad... son eficaces o ineficaces de un día para otro, con uno u otro alumno, con uno u otro profesor... y por supuesto en diferentes materias.

Podemos sugerir generalizaciones: yo no realizaría un Chat con más de 8 participantes aproximadamente, pero es posible que algún profesor/a sea capaz de obtener resultados increíbles en sesiones con 20 participantes y alguno habrá que no debería reunir a más de 4 simultáneos.

Los alumnos y profesores son personas, esto es, presentan un grado de complejidad y un nivel de diferencias infinitamente superiores a los que podemos encontrar en los tornillos. Por tanto no es posible seguir tratando de aplicar a la formación estrategias provenientes del campo de la industria.

\section{Falsas ideas previas sobre el diseño de los entornos}

Existen otras ideas equivocadas (algunas ya esbozadas) pero absolutamente generalizadas que afectan al modo como se diseñan los entornos, también conocidos como "campus virtuales". Vamos a hacer un rápido repaso a algunas de ellas:

- Diseñar un curso virtual es preparar los materiales del curso y colocarlos en Internet.

- Los campus virtuales han de contener todas las herramientas que necesita utilizar el alumno.

- Todos los cursos en un campus virtual han de responder al mismo modelo (ya comentado).

- Un campus virtual necesita de una plataforma tecnológica.

\section{Contenido versus continente}

Este es un error frecuente en muchísimos programas de formación bajo cualquier modelo: creer que la formación se reduce a la transmisión de contenidos. La idea puede parecer obvia. A fin de cuentas hoy todos hablan de competencias.

Y sin embargo a la hora de poner en marcha un sistema de EaD, y quizás por influencia del viejo modelo de la OU basado en materiales, lo primero que se piensa es en contenidos. Después se añaden las herramientas. 
Considerando la cantidad de recursos informativos disponibles en Internet, este planteamiento se traduce en un gasto innecesario de recursos que después penaliza los recursos destinados a la formación. Utilizando una terminología al uso, habría que recordar "cuál es nuestro negocio": y respondiendo en esa terminología diríamos que nuestro "negocio" es la formación y no la información. Este último corresponde a empresas editoriales, bibliotecas, centros de documentación, etc. Pero no es el "negocio" de las universidades. No deben ser "templos del saber" sino "templos del aprendizaje y la formación”.

En el análisis que realiza Kart Steffens (2006) sobre diferentes entornos de aprendizaje potenciados por la tecnología, distingue entre "Container system" versus "Content system". El segundo se distingue por estructurar el entorno alrededor del contenido a aprender, en tanto que el primero se centra en el propio entorno y los recursos que ofrece: "Container systems son sistemas de gestión del aprendizaje o la información cuyo contenido es proporcionado por el usuario. Content systems ya contienen un contenido específico". (Steffens, 2006, p. 368)

No debe extrañarnos que cada vez haya más programas docentes que ofrecen sus materiales abiertos y gratuitos, reservando sus recursos de formación para los estudiantes registrados. Quizás el caso más notable sea el del MIT: http://ocw.mit. edu/index.html

Este concepto puede verse también en la plataforma Moodle, en la opción "formato social" al diseñar un curso.

\section{Abiertos versus cerrados}

Ya se ha comentado la obsesión por colocar los materiales en entornos cerrados de modo que sólo los estudiantes puedan acceder. En ocasiones es una decisión requerida para respetar los derechos de autor de materiales reproducidos y sometidos a restricciones. Pero en muchos otros casos es un error.

De hecho posiblemente estos estudiantes encontrarían en Internet más y mejores materiales que los que les está ofreciendo el curso. El conocimiento social invade la red y compite con el conocimiento "de autor" o tradicional. Giles (2005) ha estudiado como la Wikipedia se aproxima a la Enciclopedia Británica en precisión de las entradas. 
Si alguien desea proteger de alguna manera sus derechos o evitar que otros puedan hacer negocio o manipular las propias creaciones no tiene sino que registrarlas bajo una licencia de Creative Commons $<\underline{\text { http://creativecommons.org/ }>}$

\section{Plataforma versus herramientas libres de internet}

¿Es mejor tener una herramienta o utilizar los recursos libremente disponibles en Internet?

Utilizar los recursos libres en Internet quiere decir utilizar sistemas de correo electrónico personales, colocar las webs públicas o privadas, utilizar quizás blogs de Bloger, colocar los vídeos en Youtube, listas ofrecidas por servicios gratuitos, Chat vía Skype o Messenger o ...

Algunos argumentos a favor de utilizar una plataforma:

- Control sobre los accesos (tiempos y contenidos).

- Control sobre los recursos.

- Estabilidad de los recursos ofrecidos.

- Respeto a la propiedad intelectual.

Algunos argumentos a favor de utilizar recursos abiertos:

- Desarrollo de competencias digitales útiles al terminar los estudios.

- Flexibilidad de las herramientas, e.g. el correo.

- Mayor efectividad de algunas herramientas.

- Potenciación de la acción tutorial como soporte a la evaluación frente a sistemas basados en el control de accesos o contribuciones.

Mi experiencia me muestra que la mejor solución es aquella que utiliza recursos internos y externos de acuerdo con las necesidades del momento. 


\section{UN MODELO PARA EL BLENDED LEARNING}

El análisis de las limitaciones anteriores podría llevarnos a pensar que todo el problema se reduce a un adecuado uso de los recursos. Si así lo entendiéramos estaríamos precisamente entrando en la definición más clásica de BL.

Pero en realidad, la respuesta se encuentra en un nuevo modelo, modelo quizás más cercano a las comunidades de aprendizaje, modelo en el que la presencialidad y la virtualidad se mezclan de un modo continuo.

\section{Blended Learning vs. Blended lives}

La profesora comentó a sus alumnos de 12 años:

- "Porque todos tenéis correo electrónico, claro".

Los alumnos se miraron con gestos de interrogación. La profesora, sorprendida:

- “¿No sabéis lo que es el correo electrónico?"

Nuevos gestos de duda hasta que por fin uno, más espabilado, levanta la mano:

- "Ah, sí, es eso que el Messenger te avisa que ha llegado uno".

Unos días más tarde de que una compañera me contara esta anécdota, mi hija me enseñaba este mensaje recibido de una amiga:

"I tried to connect you by MSN, but that didn't work. So now this 'oldfashioned' way;)"

Durante un tiempo había especulado con la posibilidad de que el uso preferente de sistemas de mensajería y Chat frente al correo electrónico por parte de los jóvenes tenía alguna relación con las habilidades de lectoescritura.

A partir de las anécdotas anteriores comprendí que el problema era otro: la diferencia está entre quienes "se conectan" y unos jóvenes que viven "conectados" permanentemente, bien vía Messenger, vía mensajes de móvil... Es lo que se ha dado en llamar las poderosas "redes sociales" a las que hace referencia Manuel Castells (1999).

Mientras los educadores hablamos de "blended learning", es decir, un aprendizaje en el que se mezclan recursos presenciales y virtuales, los alumnos comienzan a 
venir de otro entorno, viven "blended lives", vidas en las que se mezclan la realidad presencial y la virtual.

Mientras nosotros nos planteamos la posibilidad de introducir elementos de e-learning, y de mediante el BL optimizar los aprendizajes, los chicos de 12 años simplemente ya viven mezclando ambas realidades.

No necesitamos pedir a los alumnos que preparen un trabajo en grupo utilizando un foro o un blog: descubriremos que pronto lo harán sin que se lo pidamos. Quiero insistir en esta diferencia entre muchos estudiantes universitarios del año 2006 y los que están subiendo, que ahora tienen 12 o 15 años. Recientes estudios muestran que los jóvenes comienzan a pasar más tiempo en Internet que frente al televisor. Aunque naturalmente la situación varía según países e instituciones: por ejemplo, en Chile, la encuesta Casen 2003 indica que más de la mitad de los niños acceden al ordenador únicamente en la escuela. Si consideramos los más pobres, hablamos de que 8 de cada 10 sólo acceden a la informática vía la escuela (Casen, 2004). Es la brecha digital.

Éste es, pues, el primer punto que los profesores tenemos que tener claro: no vamos a utilizar un diseño de BL porque sea más eficaz sino porque es el modo usual de comunicarse, de acceder a la información, de gestionar nuestras redes sociales, de construir el conocimiento...

\section{Los rasgos del modelo}

No sería inteligente cometer con el BL los mismos errores que se cometieron con el e-learning. Y el primero sería tratar de introducir las herramientas sin cambiar el modelo. Pero ¿cuáles pueden ser los rasgos de ese modelo? Podemos partir del análisis efectuado anteriormente sobre el e-learning. Y estas serían algunas conclusiones:

- Diseñemos un entorno que incluye actividades, recursos con los que interactúan personas, que también interactúan entre sí. Dejemos pues de lado la idea de colocar los materiales en la red, como si la función fuera sustituir o complementar una clase magistral por una reproducción de sus contenidos en forma escrita.

- Planteemos el entorno comoun espacio en el que el sujeto desarrollecompetencias básicas como la capacidad de autorregular el aprendizaje o las digitales. 
- Ofrezcamos al alumno un entorno rico en recursos de modo que el sujeto pueda determinar sus necesidades de formación, encontrar los recursos que pueden ayudarle a solucionarlas y aplicarlos de modo efectivo.

- Proporcionemos a ese entorno de herramientas comunicativas e informativas, dotando de un carácter "tutorial" al propio entorno.

- Consideremos la importancia de los aspectos emocionales en la comunicación humana. Y valoremos especialmente lo que en este aspecto nos aportan ciertas acciones presenciales. Por ejemplo una entrevista tiene tal potencialidad en este aspecto que sería delito tratarla como un simple intercambio aséptico de informaciones. La entrevista personal es un momento privilegiado para establecer bases poderosas para un aprendizaje efectivo.

- No descuidemos, de todos modos, la potencialidad del lenguaje audiovisual.

- Dotemos a nuestro entorno de flexibilidad, de modo que profesores y estudiantes se encuentren cómodos en él, que puedan utilizarlo adaptándolo a sus necesidades y características.

- Recordemos que mucha de la información ya está en Internet o en libros y revistas: no repitamos trabajos tediosos. Centrémonos en las actividades que permitirán a nuestros alumnos desarrollar las competencias requeridas. Sólo cuando veamos que necesitará alguna información no disponible, entonces se la facilitaremos. Pero no limitemos el desarrollo de su capacidad de buscar, valorar, seleccionar, estructurar la información.

- No tratemos de ofrecer todos los recursos: existen muchas opciones en Internet. Utilizarlas no sólo nos ahorrará costos y esfuerzo, sino que además preparará a los alumnos para poder seguir haciendo uso de ellas cuando termine su formación.

Curiosamente, todos estos rasgos son compatibles con modelos didácticos muy diferentes. Por ejemplo, es posible crear un entorno BL con este modelo tanto si trabajamos por proyectos, centrados en problemas o a partir de temas o actividades. Igualmente podemos utilizarlo si nos planteamos objetivos como si nos orientamos a competencias específicas. Aunque sí que parte de una concepción del currículum flexible y centrado en el sujeto. 


\section{Diferentes modelos}

Cuando aquí planteamos un modelo para el Blended Learning nos estamos refiriendo a un modelo abierto y flexible. Es un punto de partida diferente al de otros autores que prefieren pensar en modelos muy concretos que responden a necesidades de cursos específicos. Así Valiatahn (2002) cita tres modelos:

- Aprendizaje orientado a las destrezas.

- Aprendizaje orientado a las actitudes.

- Aprendizaje orientado a las competencias.

Trabajando en esa perspectiva, es decir, en modelos diferenciados, personalmente propondría dos grandes modelos:

\begin{tabular}{|c|l|}
\hline $\begin{array}{c}\text { Modelo } \\
\text { práctico }\end{array}$ & $\begin{array}{l}\text { Basado en proyectos, problemas con entrevistas personales de evaluación y } \\
\text { seguimiento, y sesiones de grupo para contenidos específicos. Este modelo está } \\
\text { descrito por Mariona Grané (2004). }\end{array}$ \\
\hline $\begin{array}{c}\text { Modelo } \\
\text { teórico }\end{array}$ & $\begin{array}{l}\text { Basado en comunidades: sesiones de presentación en clase, lecturas y trabajo } \\
\text { con la información en la red, discusión online y presencial. }\end{array}$ \\
\hline
\end{tabular}

Y finalmente en el extremo podemos tomar la afirmación de Garrison y Kanuka (2004): "No existen dos diseños de Blended Learning idénticos".

\section{ELEMENTOS EN UN ENTORNO BL}

Es imposible pretender ser exhaustivo considerando la riqueza de recursos disponibles y que cada día aparecen en Internet. Pero al menos vamos a ver un panorama de los recursos disponibles a mediados del año 2007. Sobre todo nos puede servir más para ver cómo enfocar el uso de los recursos en entornos semipresenciales que por el uso de un recurso en concreto.

\section{Formando a grupos}

Dada la importancia que hemos visto tiene el grupo en la culminación de los estudios universitarios para muchos estudiantes, no debe extrañar la importancia 
del trabajo colaborativo en los diseños BL. Por otro lado, que el grupo es el espacio donde naturalmente es posible desarrollar la competencia 6 ("Competencias interpersonales, interculturales y sociales, y competencia cívica”) dela recomendación del Consejo de Europa sobre competencias clave para el aprendizaje permanente (Comisión, 2005).

\section{La sesión presencial: la clase}

La sesión presencial con el grupo, lo que podríamos llamar la vieja clase magistral, no necesariamente magistral por cierto, sigue siendo un recurso tremendamente valioso, quizás más, al quedar desprovisto de la necesidad de ser utilizado como vehículo de transmisión de contenidos.

Todavía muchos profesores siguen creyendo que la sesión presencial en entornos semipresenciales tiene la misma función que antes: transmitir contenidos. Eso explica también el uso masivo y el tipo de uso de las presentaciones "PowerPoint", auténtica amenaza para la docencia universitaria (Aliaga y Bartolomé, 2006).

Esto ha dejado de ser así por dos motivos:

- El diseño basado en competencias se centra en las actividades que permiten el desarrollo de competencias y no en la transmisión de contenidos aún cuando conocer sea parte del proceso de adquisición de competencias.

- Existen recursos mucho más adecuados para transmitir la información.

Por ello la sesión presencial debe centrarse en tareas como:

- Presentar de modo global un tema.

- Dar pautas para un trabajo.

- Incentivar a los alumnos, ayudarles a encontrarse motivados.

- Mostrar la relación de un tema con otros.

- Presentar los elementos fundamentales de un tema de un modo sucinto.

- Sugerir aspectos importantes a estudiar.

- Generar dinámicas de grupo que ayuden al aprendizaje.

- Realizar tutoría en grupo. 
- Supervisar actividades individuales o en grupo simultáneas.

- Presentar paquetes pequeños y precisos de información (corta duración).

- Mostrar la aplicación práctica de un aspecto teórico.

- Presentar dispositivos, ejemplos, experiencias, ... (duración media, preferible en grupos pequeños).

Y por supuesto es de nuevo la gran ocasión para retomar el agrupamiento flexible de los años sesenta y plantearnos que en ocasiones podemos trabajar con un grupo de 150 alumnos, muchas veces con grupos de 30, y también con grupos pequeños.

Los recursos comunicativos virtuales: correo, foros, listas, chats, videconferencias

Hace muchos años que una serie de recursos comunicativos han sido utilizados para potenciar el trabajo en grupo en los programas de e-learning (Cabero y Ot. 2004; Hiltz, 1997; Meyer, 2003). De ahí pasaron a los sistemas BL. Algunos de ellos se caracterizan por ser recursos que permiten la comunicación punto a punto, por ejemplo el correo electrónico.

Otros permitían la comunicación no presencial en el grupo y son sobradamente conocidos el Chat, por lo que hace a una comunicación poco estructurada y de mensajes cortos en tiempo real, y los foros y listas de discusión por lo que hace a una conversación estructurada, con capacidad para la exposición detallada de ideas, y a la que no se necesita acceder de modo simultáneo.

En general la mayor limitación de estos instrumentos ha sido su dependencia de la comunicación escrita, sus limitaciones por lo que se refiere a mensajes no verbales con lo que implica en relación a los aspectos emocionales, y la falta de dinámicas de grupo adecuadas que pudieran compararse con las utilizadas en las reuniones en grupo presenciales.

También es verdad que en ocasiones han sido criticadas por la falta departicipación cuando ésta no era menor de la que podríamos encontrar en una reunión con el grupo de clase.

Se han experimentado diferentes dinámicas y no es éste el sitio para extenderse en ellas, pero querría hacer notar la importancia de conectar la discusión virtual con 
el diálogo presencial, así como del papel del profesor entendido como un animador del grupo.

Hay que decir que el Chat se está viendo potenciado últimamente por la videoconferencia y la audioconferencia. No son recursos nuevos, ciertamente. Pero algunas soluciones técnicas, como Skype que permiten ahora plantearse el recurso con elevada calidad y bajo costo.

Creo que una explicación a algunos problemas encontrados por los profesores cuando han puesto en marcha foros y Chats ha sido que han entendido que "ese" era el trabajo en grupo que proponían. No es así: los foros, etc. son meros instrumentos de comunicación, al servicio de la actividad que se realice, sea una acción tutorial, sea un proyecto en grupo.

Por ejemplo, la actividad a diseñar no debería ser: "Vamos a organizar un foro", sino, "Vamos a crear un informe en grupo" o "Vamos a analizar una situación en grupo" o... Y para esa tarea utilizaremos diferentes recursos, incluidos recursos comunicativos como el correo, el foro o el Chat.

Los nuevos modos de participación y construcción de la información

Existen dos nuevas herramientas de construcción y distribución de la información tremendamente poderosa y que se encuadran en lo que podría llamarse la Web 2.0: los blogs y las wikis. No es éste el lugar donde describirlos y analizarlos, pero también es necesario realizar algunas precisiones. Se supone que el lector ya los conoce. En caso contrario puede recurrir a la Wikipedia:

- $\quad$ http://es.wikipedia.org/wiki/Blog

- $\quad$ http://en.wikipedia.org/wiki/Wiki

A diferencia de foros y Chats, éstas no son herramientas de comunicación, aunque ésta sea posible a través de los comentarios a las entradas en los blogs, o de la sección "discusión" de una wiki.

Los blogs son básicamente portales de conocimiento en el marco de un "conocimiento o saber distribuido", donde ningún punto de acumulación de información convertida en ideas se presenta como superior o exclusivo, sino como parte de una red no necesariamente competitiva sino colaborativa de creación cognitiva. Así, frente a los portales de periódicos, centros de información o 
televisiones, los blogs parten de una estructura horizontal en forma de rizoma en la que la información y el conocimiento fluyen y se construyen a través de todas las raíces subterráneas que las comunican: cada pequeña brizna de césped que vemos en la superficie no es sino parte de la "blogosfera".

El uso de blogs en cursos responde básicamente a dos modelos: en el primero cada estudiante o grupo posee su propio blog, y éste actúa como equivalente del portafolio digital, así como para permitir los intercambios entre sujetos y el desarrollo de competencias en aspectos como el acceso a la información o la capacidad de expresión escrita.

En el segundo modelo un único blog actúa como eje vertebrador del curso, actuando como sustituto de una plataforma o de una web. Parece que una de las razones por la que varios profesores han escogido esta opción es por la extraordinaria facilidad de creación frente a las otras soluciones. Es obvio que también presenta ventajas como el "descubrimiento progresivo" del curso conforme se van introduciendo las entradas, el carácter único de cada curso (cada año se crea uno diferente) o incluso la posibilidad de convertir un curso en un proceso continuo en el que también participan alumnos de años anteriores.

Pero también hay que recordar a los profesores entusiastas que igual que un blog nos permite analizar el trabajo del alumno que lo crea de un modo más transparente de lo que nunca pudo hacerse con los viejos trabajos en papel o pdf, la misma transparencia afecta al trabajo del profesor: un blog de curso nos muestra con qué frecuencia el profesor o los alumnos actualizan entradas, qué participación se da a través de los comentarios, en que medida las intervenciones reflejan un conocimiento de la realidad mediante enlaces, etc.

Finalmente el blog representa un modo democrático de compartir y acceder a la información. Se conciben en el marco de una nueva forma de conocer, en la que sindicarse a blogs mediante agregadores es la vía para estar informado. En ese contexto, un profesor situado en el paradigma de la autoridad magistral y la comunicación vertical podría encontrar fácilmente problemas.

Las wikis son un recurso completamente diferente: se trata de documentos de creación social, con diseño hipertextual y carácter dinámico. Veamos algunas diferencias: 
- Un blog posee una estructura temporal mientras la wiki posee una estructura ligada al tema.

- Una entrada de un blog no cambia, permanece igual aunque enriquecida con comentarios añadidos. Una página de una wiki cambia en el tiempo.

- Los textos en un blog son textos lineales con enlaces hacia fuera. Los textos en una wiki son construidos en forma de paquetes enlazados de información, y también poseen enlaces hacia fuera. Podemos decir que aún cuando ambos se conectan al Hipertexto que es la WWW, sólo la segunda posee en sí también una estructura hipertextual.

- En un blog hay un autor o autores propietarios que crean entradas. Los visitantes están autorizados a escribir comentarios. En la wiki todos los participantes son autores que pueden modificar los textos (no existe diferencia entre "entradas" y "comentarios"). Naturalmente, este punto varía en función de la configuración de los documentos, tanto blogs como wikis.

Contra lo que sucedía con foros y Chats, aquí blogs y wikis pueden considerarse el objeto del trabajo en grupo, en tanto en cuanto formatos de creación de conocimiento.

No debe pensarse que el mundo virtual oculta el real. Los encuentros personales (F2F por "cara a cara") entre los participantes en blogs tienen incluso su propia denominación en su argot: "Beers \& blogs", literalmente, "cervezas y blogs", o si se prefiere; "¿qué tal si mañana nos vemos cara a cara y tomamos unas cervezas?”.

\section{Información y conocimiento}

Los recursos para distribuir la información y el conocimiento son sobradamente conocidos. Pero han aparecido últimamente algunos novedosos que merecen ser comentados.

La información también es audiovisual: Youtube y los podcasts

Aunque siempre ha sido posible introducir elementos audiovisuales, sólo recientemente esta opción se plantea como algo fácil y asequible. Existen dos grandes opciones: Youtube y los podcasts. 
YouTube no es sino un sistema para compartir vídeos en Internet y existen otros. (http://www.youtube.com/)

Sin embargo la facilidad de uso lo ha convertido de pronto en un recurso valioso tanto para el profesor como para el alumno. Tradicionalmente ambos encontraban dos grandes obstáculos al uso del vídeo:

- La dificultad de producción.

- La dificultad de almacenamiento y distribución.

La primera ha quedado en gran medida resuelta gracias al vídeo digital. Hoy es posible preparar un pequeño clip de 1 minuto sin más que una webcam y el propio notebook.

Pero la segunda no era tan sencilla. El tamaño de los ficheros hacía desaconsejable enviarlos por correo. En muchos campus virtuales se excedían del tamaño máximo permitido para ser enviados al profesor o ser colgados.

Subir un vídeo a YouTube es fácil. Pero además no es necesario "verlos" en YouTube. Basta incluir el código "EMBED" para que el vídeo se visione en nuestra página, aunque eso sí, con la marca de agua de Youtube, pero sin toda la parafernalia de la página.

Descargar un vídeo de Youtube también es fácil mediante los recursos que han aparecido como el "VideoDownloader" en: http://javimoya.com/blog/youtube.php

Los podcasts representan una solución completamente diferente (para información sobre los podcasts ver la wikipedia):

- Aunque relativamente fácil, no todos los profesores la encuentran sencilla.

- El concepto de compartir gratis tiene aquí un peso mucho mayor, al menos por ahora: los vídeos o ficheros de audio son descargados en el equipo del receptor.

- Representan algo así como una "radio o televisión personal" al estar ligados si se desea al uso de dispositivos de reproducción portátil.

- Aceptan formatos de duración media y larga. En ese sentido algunos profesores los han percibido como cercanos a su modelo tradicional de enseñanza magistral. De hecho suelen estar más vinculados a las acciones presenciales. 
Precisamente este último aspecto es el que más me preocupa. Mientras hay profesores que los han integrado en su sistema docente como lo que son, sistemas de distribución de información audiovisual en el marco de entornos de aprendizaje ricos en recursos, hay otros que los han visto como un "sustituto" de la clase presencial.

Es sorprendente encontrar todavía reacciones de rechazo ante el audiovisual: una profesora de Química Inorgánica aseguraba que no necesitaba para nada el audiovisual. Estos son algunos de los ejemplos de lo que podemos hacer con pequeños clips de vídeo de muy pocos minutos, fáciles de producir:

- Mostrar una práctica antes de hacerla.

- Mostrar un ejemplo de la vida real.

- Plantear un problema.

- Mostrar de modo intuitivo una idea, demostrar gráficamente un problema.

- Mostrar las personas que citamos, cómo son, cómo hablan... (eso convierte áridos nombres en personajes reales, y facilita el aprendizaje).

- Mostrar buenas prácticas... y malas prácticas.

- Utilizar como punto de partida para una discusión (que luego puede ser continuada de modo virtual).

Y muchas otras según los casos. El audiovisual es poderoso. Y ahora además accesible.

Lineal versus hipertextual: ficheros pdfy páginas web hipertextuales

Algunos profesores parece que desconozcan que es posible construir documentos no lineales. Se limitan a proporcionar a sus alumnos abundantes documentos en formato pdf.

Pero también hay quien cree que el hipertexto es lo mejor, lo único.

No es así. Aunque aquí no tratemos el tema en detalle, diremos que en la línea planteada, la información en soporte textual (sea papel o electrónica, sea lineal o hipertextual) debe ofrecerse de modo diverso para atender a las necesidades de alumnos diferentes. 
Quizás un buen ejemplo lo podemos tomar de los programas informáticos. ¿Qué nos ofrecen para aprender a utilizarlos?

- Una guía “Quick Start”: documento lineal corto que introduce a aspectos clave. Adecuado para comenzar, para alumnos que lo desconocen todo.

- Un tutorial: adecuado para alumnos con estilo de aprendizaje no autónomo, o con poca iniciativa.

- Un manual: es un documento lineal, largo, estructurado, que el sujeto puede seguir o en el que puede consultar. Provisto de índices de acceso. Adecuado para alumnos novicios, pero también para expertos, cuando desconocen totalmente un aspecto o cuando desean conocerlo en profundidad.

- Una ayuda online: un documento hipertextual que nos permite encontrar informaciones concretas y responder a necesidades específicas. Además nos permite navegar por la información sin perder tiempo en aspectos que no nos interesan. Pero requiere una cierta habilidad para navegar sin perderse o despistarse, y un cierto conocimiento del tema para optimizar la navegación sin falsos viajes. Alumnos expertos.

- Una ayuda sensible al contexto: en cada momento está la pequeña frase que nos ayuda a continuar sabiendo donde estamos.

- Una lista de discusión: un sistema de tutoría social en el que aclarar dudas (ver este y los siguientes apartados en el punto referido a tutoría).

- Una lista de preguntas frecuentes: la solución para los obstáculos que se repiten año tras año.

- Cursos y talleres presenciales.

- Sistemas de atención a consultas personales.

Todos estos recursos podemos incorporarlos a nuestros cursos. Y dejemos que cada alumno vaya aprendiendo y accediendo a la información en el modo que mejor se adapte a sus características. Pero no pretendamos prepararlo todo nosotros: otros muchos profesores y profesoras han colocado antes en Internet materiales y recursos valiosos: aprovechémoslos y compartamos los que tengamos que preparar. 
Pero recordemos que los contenidos informativos NO son el entorno de formación: forman parte del mismo y están al servicio de las actividades que hemos diseñado.

\section{Aprendizaje autónomo}

El uso de recursos para el aprendizaje autónomo es una de las grandes aportaciones de Internet. Puede ser en forma de recursos públicos o de pago, tipo simulaciones, tutoriales, casos, problemas, ejercicios, etc. o bien encapsulados en forma de Objetos de aprendizaje.

La flexibilidad en los recursos: los Objetos de aprendizaje

Los "Objetos de aprendizaje" no son un fin en sí mismo, sino un modo de distribuir los recursos de aprendizaje de modo que resulte cómodo encontrar el que mejor se adecue a las necesidades particulares.

Una clarificación excelente sobre los Objetos de aprendizaje, concepto, estructura y aplicación, pueden verse en el artículo de Jordi Adell (2004).

La clave no es que sea o no un "Objeto de aprendizaje”. La clave es el diseño del objeto.

La flexibilidad en el modelo: de la ejercitación a las simulaciones

Tanto si escogemos recursos para el autoaprendizaje como si trabajamos con Objetos de aprendizaje (no informativos) podemos considerar 4 modelos de programas (Bartolomé, 1998), que nos permitirán trabajar de un modo más preciso las competencias que nos interesan:

- Programas de ejercitación: la práctica y la repetición son la base de los aprendizajes de destrezas. No serán el único elemento de un curso, pero si existe la necesidad de desarrollar una habilidad específica, posiblemente ésta necesitará de práctica y de ejercicios. Los sistemas de corrección automática no son necesariamente los mejores: la autocorrección puede resultar mucho más eficaz para un aprendizaje duradero.

- Tutoriales: un profesor puede guiar un aprendizaje específico mediante un programa tutorial en el que ha previsto los errores más frecuentes que puede 
encontrar y como guiarle para solucionarlos. Son muy prácticos si recordamos que no son meros textos informativos como algunos creen, sino que deben incluir la participación del sujeto y una guía que tenga en cuenta sus respuestas.

- Casos y problemas: el aprendizaje orientado a la resolución de casos y problemas posee una gran capacidad incentivadota. Las Webquest pueden considerarse una variante de este diseño. Y además son fáciles de desarrollar.

- Simulaciones: en general son recursos costosos aunque también es posible preparar sencillas simulaciones con ciertos conocimientos de programación. Existen abundantes en Internet.

La siguiente tabla nos mostraría las competencias que permiten desarrollar cada modelo de un modo más adecuado.

\begin{tabular}{|l|l|l|}
\hline Modelo & Competencias transversales & Competencias específicas \\
\hline Ejercitación & & Destrezas simples \\
\hline Tutoriales & & Conocimientos básicos \\
\hline Casos y problemas & Búsqueda de Información & Conocimientos profundos \\
\hline Simulaciones & Toma de decisiones & Destrezas complejas \\
\hline
\end{tabular}

\section{Tutoría y Evaluación}

No es necesario repetir aquí que la tutoría debe ser entendida como un entorno que incluye recursos ya citados. Una tabla resumen sería:

- Presenciales

- Entrevistas personales

- Sesiones en pequeño grupo

- Sesiones en grupo de clase

- Virtuales

- Páginas de preguntas frecuentes

- Listas y foros de dudas

- Correo asistido y correo inteligente 
El diseño del entorno no elimina al tutor, sino que por el contrario realza su papel como orientador, despojándolo del papel de "contestador automático de preguntas", en la línea de lo que solicitan los estudiantes según la encuesta de Valiathan (2002).

\section{Un entorno para la formación}

Hablar de Blended Learning es hablar de una visión rica, flexible y abierta de lo que debe ser el entorno en el que se produzca el aprendizaje. Los profesores no podemos diseñar el aprendizaje, ya que esa es una tarea del alumno. Pero podemos ofrecerle un entorno en el que el estudiante pueda desarrollar las competencias de acuerdo con sus necesidades y, sus habilidades y conocimientos previos.

Hablar de Blended Learning es hablar de entornos de formación en una sociedad que se caracteriza por tal desarrollo de las TIC que realidad y virtualidad se mezclan. De hecho, es discutido el concepto de virtualidad, pues no es sino la realidad mediada de modo diferente. Entre una conversación con un alumno en el aula, por videoconferencia o en el Chat sólo cambia el medio a través del cual se produce la comunicación. Por eso Blended Learning no es sino no ponerle límites a los medios que utilizamos en nuestra comunicación.

Así, en definitiva, hablar de Blended Learning es hablar de comunicación, de una comunicación mediada de formas muy diferentes, que se beneficia de la riqueza de códigos y tecnologías para potenciar la comunicación. Pues la Educación, como decía el Dr. Rodríguez Diéguez, no es sino un tipo especial de comunicación. 


\section{REFERENCIAS BIBLIOGRÁFICAS}

Adell, J. (2004). Nuevas tecnologías en la formación presencial: del curso online a las comunidades de aprendizaje. Qurriculum: Revista de teoría, investigación y práctica educativa, 17, 57-92.

Aiello, M.; Willem, C. (2004). El Blended Learning como práctica transformadora. Pixel-Bit.Revista de Mediosy Educación, 23,21-26. [enlínea] Disponibleen:http:// www.sav.us.es/pixelbit/articulos/n23/ n23art/art2302.htm [consulta 2007, 15 de marzo]

Aliaga, F.; Bartolomé,A. (2006). Elimpacto de las nuevas tecnologías en Educación, en: Escudero, T.; Correa, A. Investigación en Innovación Educativa, 55-88. Madrid: La Muralla.

Bartolomé, A. (1998). Sistemas multimedia en Educación, en: Pablos, J.; Jiménez, J. Nuevas Tecnologías. Comunicación Audiovisual y Educación. Barcelona: Cedecs. 149-176. [en línea] Disponible en: http://www.lmi.ub.es/personal/ bartolome/articuloshtml/g8 multimedia/index.html [consulta 2007, 15 de marzo]

Bartolomé, A. (2001). Universidades en la Red. ¿Universidad presencial o virtual?, en: Crítica, LII (No 896), 3438. [en línea] Disponible en: http:// www.lmi.ub.es/personal/bartolome/ articuloshtml/bartolomeSPcritica02.pdf [consulta 2007, 15 de marzo]

Bartolomé, A. (2004). Blended Learning. Conceptos básicos. Pixel-Bit. Revista de Medios y Educación, 23, 7-20. [en línea] Disponible en: http://www.lmi.ub.es/ personal/bartolome/articuloshtml/o4 blended learning/documentacion/1 bartolome.pdf [consulta 2007, 15 de marzo]

Bartolomé, A.; Aiello, M. (2006). Nuevas tecnologías y necesidades formativas.
Blended Learning y nuevos perfiles en Comunicación Audiovisual, en Telos, 67, Abril-Junio 2006, cuaderno central. [en línea] Disponible en: http://www. campusred.net/telos/articulocuaderno. asp?idarticulo $=2 \&$ rev $=67 \quad$ [consulta 2007, 15 de marzo]

Brennan, M. (2004). Blended Learning and Business Change. Chief Learning Officer Magazine. Enero 2004 [en línea] Disponible en: http://www.clomedia. com/content/anmviewer.asp? $\mathrm{a}=349$ [consulta 2007, 15 de marzo]

Brodsky, M. W. (2003). Four Blended Learning Blunders and How to Avoid Them. Learning Circuits, Noviembre 2003 [en línea] Disponible en: http:// www.astd.org/ASTD/Publications/ LearningCircuits/2003/nov2003/ elearn.html [consulta 2007, 15 de marzo]

Cabero, J.; Ot. (2004). Las herramientas de comunicación en el aprendizaje mezclado. Pixel-Bit. Revista de Medios y Educación, 23, 27-41. [en línea] Disponible en: http://www.sav.us.es/ pixelbit/articulos/n23/n23art/art2303. htm [consulta 2007, 15 de marzo]

Casas, M.; Stojanovic, L. (1999). De Vancouver (1982) a Viena (1999): La Educación a Distancia Toma Nuevos Rumbos. RIED Revista Iberoamericana de Educación a Distancia, 2 (2), Diciembre de 1999. [en línea] Disponible en: http://www.utpl. edu.ec/ried/index.php?option $=$ com c ontent\&task $=$ view\&id $=125 \&$ Itemid $=53$ [consulta 2007, 15 de marzo]

Casen (2004). Encuesta de Caracterización socioeconómica nacional. Casen 2003. Gobierno de Chile. Mideplan. [en línea] Disponible en: http://biblioteca. mineduc.cl/documento/CASEN EDUCACIONAL (sintesis ejecutiva). pdf [consulta 2007, 15 de marzo] 
Castells, M. (1999). Internet y la Sociedad Red. Lección inaugural del programa de doctorado sobre la sociedad de la información y el conocimiento (UOC). [en línea] Disponible en: http://www. livros.online.pt/ideias/pdf/IOP Castells Internetylasociedaddered.pdf [consulta 2007, 15 de marzo]

Cilia, W.; Aiello, M.; Bartolomé, A. (2006). Self-Regulated Learning and New Literacies: an experience at the University of Barcelona. European Journal of Education, Vol. 41, No. 3/4, 437-452.

Coaten, N. (2003). Blended e-learning. Educaweb, 69. 6 de octubre de 2003. [en línea] Disponible en: http:// www.educaweb.com/esp/servicios/ monografico/formacionvirtual/1181076. asp [consulta 2007, 15 de marzo]

College Board (2001). Trends in College Pricing 2001. Washington D. C.

Comisión de las comunidades Europeas (2005). Recomendación del Parlamento Europeo y del Consejo sobre las competencias clave para el aprendizaje permanente. $\mathrm{COM}(2005) 548$ final. [en línea] Disponible en: http://ec.europa. eu/education/policies/2010/doc/ keyrec es.pdf [consulta 2007, 15 de marzo]

Delestre, N.; Pétuchet, J.; Barry-Gréboval, C. (1999). Why to use a dynamic adaptative hypermedia for teaching an how to design it?, en: De Bra, P.; Leggett, J. (Eds.) WebNet'99 World conference on the World Wide Web and Internet, 277282, Norfolk (VA): AACE.

García Aretio, L. (1999). Historia de la Educación a Distancia. RIED - Revista Iberoamericana de Educación a Distancia, 2 (1), Junio de 1999. [en línea] Disponible en: http://www.utpl.edu.ec/ ried/index.php?option $=$ com content\&t ask=view\&id $=274 \&$ Itemid $=53$ [consulta 2007, 15 de marzo]
Garrison, D.; Kanuka, H. (2004). Blended learning: Uncovering its transformative potential in higher education. Internet and Higher Education 7, 95 - 105 [en línea] Disponible en: http://www. sciencedirect.com/ [consulta 2007, 15 de marzo]

Giles, J. (2005). Internet encyclopaedias go head to head. Nature, Special report 14 Dic. 2005. [en línea] Disponible en: http://www.nature.com/ news/2005/051212/full/438900a.html [consulta 2007, 15 de marzo]

Grané,M.(2004).Comunicación Audiovisual, una experiencia basada en el blended learning en la universidad. El Blended Learning como práctica transformadora. Pixel-Bit.RevistadeMedios y Educación, 23. [en línea] Disponible en: http:// www.sav.us.es/pixelbit/articulos/n23/ n23art/art2308.htm [consulta 2007, 15 de marzo]

Gutiérrez, J.; Pérez, T. (2001). Sistemas hipermedia adaptativos, en: Ortega, M.; Bravo, J. (Eds.) Sistemas de interacción Persona-Computador, 159-179. Cuenca: Ediciones de la Universidad de CastillaLa Mancha.

Heterick, B.; Twigg, C. (2003). The Learning MarketSpace. February 2003. The National Center for Academic Transformation [en línea] Disponible en: http://www.center.rpi.edu/ Newsletters/Febo3.html [consulta 2007, 15 de marzo]

Hiltz, S. R. (1997). Impacts of college level courses via synchronous learning networks: Some preliminary results. Journal of Asynchronous Learning Networks, 1 (2) [en línea] Disponible en: http://www.aln.org/alnweb/journal/ jaln-voliissue2.htm [consulta 2007, 15 de marzo]

Jobbings, D. (2005). Exploiting the educational potential of podcasting. Recap Russell Educational Consultancy and Productions. United Kingdom, 
Julio. [en línea] Disponible en: http:// www.recap.ltd.uk/articles/podguide. html [consulta 2007, 15 de marzo]

Johnson, C. (2001). A survey of current research on online communities of practice. Internet and Higher Education 4,45-6o. [enlínea] Disponible en:http:// dissertation.martinaspeli.net/papers/ communities-of-practice/johnson-2001a-survey-of-current-research-on-onlinecommunities-of-practice/johnsononline-cop.pdf [consulta 2007, 15 de marzo]

Leão, M. B.; Bartolomé, A. (2003). Multiambiente de aprendizagem: a integração da sala de aula com os laboratórios experimentais e de multimeios. Revista Brasileira de Tecnologia Educacional. Anos XXX/ XXI, No. 159/160, 75-80. Marzo.

Marsh, G. E.; Mcfadden, A. C.; Price, B. J. (2003). Blended Instruction: Adapting Conventional Instruction for Large Classes. En Online Journal of Distance Learning Administration, (VI), No IV, Winter [en línea] Disponible en: http:// www.westga.edu/ distance/ojdla/ winter64/marsh64.htm [consulta 2007, 15 de marzo]

Meyer, K. A. (2003). Face-to-face versus threaded discussions: The role of time and higher-order thinking. Journal of Asynchronous Learning Networks, 7 (3), $55-65$.

Oliver, M.; Trigwell, K. (2005). Can 'Blended Learning' Be Redeemed? E-learning, 2 (1), 17-26. [en línea] Disponible en: http://www.wwwords.co.uk/pdf/ validate.asp?j=elea\&vol=2\&issue $=1 \& y e a$ $\underline{\mathrm{r}=2005 \& \text { article }=3 \text { Oliver ELEA } 21}$ web [consulta 2007, 15 de marzo]
Pascual, M. P. (2003). El Blended learning reduce el ahorro de la formación on-line pero gana en calidad. Educaweb, 69. 6 de octubre. [en línea] Disponible en: http:// www.educaweb.com/esp/servicios/ monografico/formacionvirtual/1181108. asp [consulta 2007, 15 de marzo]

Salinas, J. (1999). ¿Qué se entiende por una institución de educación superior flexible? Comunicación presentada en Congreso Edutec 99. NNTT en la formación flexible y a distancia, 14 a 17 de septiembre, Sevilla [en línea] Disponible en: http://tecnologiaedu. us.es/bibliovir/pdf/gte35.pdf [consulta 2007, 15 de marzo]

Schunk, D. H.; Zimmerman, B. J. (1998). SelfRegulated Learning: From Teaching to Self-Reflective Practice. New York: Guilford Press.

Sindicatura de Comptes de Catalunya (2006). Informe 6/2006. Agregado de las Universidades públicas de Cataluña. Ejercicio 2003. [en línea] Disponible en: http://www.sindicatura.org/pdfs/o6 o6 es.pdf [consulta 2007, 15 de marzo]

Steffens, K. (2006). Self-Regulated Learning in Technology-Enhanced Learning Environments: lessons of a European peer review. European Journal of Education, 41, $\mathrm{N}^{\circ}$ 3/4, 2006, 353-379. [en línea] Disponible en: http://www. blackwell-synergy.com/doi/pdf/10.1111/ j.1465-3435.2006.00271.x [consulta 2007, 15 de marzo]

Valiathan, P. (2002). Blended Learning Models. [en línea] Disponible en: http://www.learningcircuits.org/2002/ aug2002/valiathan.html [consulta 2007, 15 de marzo] 


\section{PERFIL ACADÉMICO Y PROFESIONAL DEL AUTOR}

Antonio Bartolomé Pina es Doctor en Filosofía y Ciencias de la Educación, profesor de Comunicación Audiovisual, especialista en Tecnología Educativa, su trabajo se centra en el diseño y desarrollo de entornos de aprendizaje potenciados por la tecnología y en el Vídeo digital. Ha dirigido numerosos proyectos europeos de investigación desde 1990, autor de unos veinte libros y más de un centenar de artículos. Profesor en la Universidad de Barcelona, ha sido también profesor invitado en diferentes universidades latinoamericanas (PUC, Monterrey, Salta, ...).

E-mail: abartolome@ub.edu

DIRECCIÓN DEL AUTOR

Amadeu Vives, 8

o8320 El Masnou

España

Fechas de recepción del artículo: 17/10/07

Fechas aceptación del artículo: 10/01/08 